\title{
Space-Time Symmetries in Gauge Theories
}

\author{
P. Forgács and N. S. Manton \\ Laboratoire de Physique Théorique de l'Ecole Normale Supérieure, ${ }^{\star}$ F-75231 Paris Cedex 05, France
}

\begin{abstract}
A general definition of symmetries of gauge fields is proposed and a method developed for constructing symmetric fields for an arbitrary gauge group. Scalar fields occur naturally in the formalism and the pure gauge theory reduces to a Higgs model in lower dimensions.
\end{abstract}

\section{Introduction}

In the study of solitons (instantons, monopoles, vortices) in non-abelian gauge theories, space-time symmetry plays an important role. In the case of monopoles, the only solutions which are explicitly known are those which generalize the 't Hooft-Polyakov monopole [1], which is spherically symmetric. The BPST single instanton solution of the SU(2) self-duality equations [2] exhibits fourdimensional rotational symmetry, and Witten's more general multi-instanton solutions still have three-dimensional rotational symmetry [3].

The technique used in all these cases is to find an ansatz for the gauge fields and Higgs fields, possessing the desired symmetry, to insert into the field equations. In the case of 3-D spherical symmetry, for example, such an ansatz is required to be invariant under the combined rotation generated by $\mathscr{J}_{i}$,

$$
\mathscr{J}_{i}=\mathscr{L}_{i}+\mathscr{S}_{i}+\mathscr{T}_{i},
$$

where $\mathscr{L}_{i}$ is the orbital angular momentum, $\mathscr{S}_{i}$ the spin, and $\mathscr{T}_{i}$ a generator of an $\mathrm{SO}(3)$ subalgebra of the gauge group [4]. We see that this technique involves embedding the symmetry group into the gauge group, which would also be the case for more general symmetries.

In this article we completely reconsider the question of symmetries in gauge theories. A set of symmetries is a group of motions of the underlying space-time manifold, with which we associate transformations of the fields defined on it. For a scalar field, this transformation would be just a shift of the argument. A gauge field, $A_{\mu}$, possesses this group of symmetries if the appropriate transformation only has the effect of a gauge transformation on $A_{\mu}$, hence leaving physical, gauge invariant quantities the same. We formulate this mathematically,

* Laboratoire propre du C.N.R.S. associé à l'Ecole Normale Supérieure et à l'Université de Paris Sud 
for infinitesimal motions, in terms of Lie derivatives. We have tried to make this paper almost self-contained, but for further details we refer the reader to [5].

In fibre bundle language, Bergmann and Flaherty have already proposed a general definition of a single symmetry in gauge theories in terms of a Lie derivative but they have not considered several symmetries simultaneously [6]. An alternative general approach, developed by Harnad et al., is to consider finite symmetry operations, e.g. finite rotations, which are associated with finite gauge transformations [7]. This reduces to Lie derivative conditions in the infinitesimal limit. We adopt the first of these approaches as our starting point. We obtain a set of symmetry equations which can be solved completely, as differential equations, but there remain certain algebraic constraints to be satisfied, relating the Lie algebras of the symmetry and gauge groups. An important step in the calculation requires the embedding of a subgroup of the symmetry group into the gauge group, but not the whole symmetry group. Our class of symmetric gauge fields is therefore considerably larger than the class obtained using previous techniques, and of course includes them. The symmetric gauge fields are characterized by several arbitrary functions, which can be interpreted as gauge fields and scalar fields in a lower dimensional theory. If the metric of the underlying space-time manifold has the same symmetries as the gauge fields, the Yang-Mills action reduces to that of a gauge theory with Higgs fields in lower dimensions. In general, the resulting gauge group is only a subgroup of the initial one, and the new, lower dimensional space can be curved, even if the original space is flat (or vice versa). The scalar fields have quartic interactions, and a global flavour symmetry in general, with a flavour group closely related to the original space-time symmetry group.

Witten established these interesting consequences of symmetries in gauge theories in a special case [3]. The details are important, so we summarize his results. His ansatz is for SU(2) gauge fields $A_{\mu}$ in 4-D Euclidean space, with 3-D spherical symmetry and arbitrary time dependence. For later convenience, we give his ansatz in spherical polar coordinates $(t, r, \theta, \varphi)$ and in the so-called abelian gauge, where $A_{t}^{a}, A_{r}^{a}$ have non-zero components only for $a=3$. The field components are:

$$
\begin{aligned}
& A_{t}^{a}=\left(0,0, A_{0}\right), \\
& A_{r}^{a}=\left(0,0, A_{1}\right), \\
& A_{\theta}^{a}=\left(-\phi_{1},-\phi_{2}, 0\right), \\
& A_{\varphi}^{a}=\left(\phi_{2} \sin \theta,-\phi_{1} \sin \theta,-\cos \theta\right),
\end{aligned}
$$

where $A_{0}, A_{1}, \phi_{1}, \phi_{2}$ are arbitrary functions of $t$ and $r$. Now, the action is

$$
\mathscr{L}=\frac{1}{4} \int d^{4} x \sqrt{\operatorname{det} h_{\mu \nu}} F_{\mu \nu}^{a} F_{\sigma \tau}^{a} h^{\mu \sigma} h^{\nu \tau},
$$

where $h^{\mu v}$ is the flat metric in polar coordinates

$$
h^{\mu \nu}=\operatorname{Diag}\left(1 ; 1 ; \frac{1}{r^{2}} ; \frac{1}{r^{2} \sin ^{2} \theta}\right) .
$$

Since, for the ansatz (1.2), the Lagrangian is independent of the polar angles, 
after integration the action reduces to

$$
\mathscr{L}=8 \pi \int_{-\infty}^{\infty} d t \int_{0}^{\infty} d r g^{1 / 2}\left[\frac{1}{8} F_{i j} F^{i j}+\frac{1}{2}\left(D_{i} \phi_{m}\right)\left(D^{i} \phi_{m}\right)+\frac{1}{4}\left(1-\phi_{1}^{2}-\phi_{2}^{2}\right)^{2}\right],
$$

where

$$
\begin{aligned}
& F_{i j}=\partial_{i} A_{j}-\partial_{j} A_{i}, \quad i, j=0,1, \\
& D_{i} \phi_{m}=\partial_{i} \phi_{m}+\varepsilon_{m n} A_{i} \phi_{n}, \quad m, n=1,2, \quad x^{0}=t, \quad x^{1}=r
\end{aligned}
$$

and we raise the $i, j$ indices with $g^{i j}=r^{2} \delta^{i j}$. This is the action in curved space for a two-dimensional abelian Higgs theory. The curvature scalar corresponding to $g^{i j}$ is $\mathbf{R}=-2$, so the theory is defined on a two dimensional surface of constant negative curvature. As we can see, imposing spherical symmetry on a 4-D pure SU(2) gauge theory leads to some surprises: the dimensional reduction to curved space, the appearance of Higgs fields, and the reduction of the gauge group to $\mathrm{U}(1)$.

The general methods developed in this article for constructing symmetric gauge fields lead to an understanding of these features, and we shall conclude with a reanalysis of Witten's ansatz.

Related to these ideas is the development of dimensional reduction techniques in theories of gravity. Originally proposed as a method of physically interpreting 26- and 10-dimensional dual models [8], and later applied to the physical interpretation of classical gravity in more than 4-D [9], the recent application has been to the construction of extended supergravity theories in 4-D [10]. The dimensional reduction is achieved, as in gauge theories, by imposing symmetries on the fields in the extra dimensions.

\section{Symmetries of Gauge Fields}

Since in our definition of symmetry the concept of a Lie derivative plays a fundamental role, we give an elementary description of it. Let $\phi$ be a mapping of a D-dimensional manifold $\mathscr{M}$ onto itself

$$
\phi: \mathscr{M} \rightarrow \mathscr{M} ; \quad x \mapsto \bar{x}
$$

The mapping $\phi$ could define one of the possible motions in a group of symmetries. To deal with general tensor fields, we need to consider the coordinate transformation associated with $\phi\left(x^{\mu} \rightarrow x^{\mu^{\prime}}\right)$ which gives the point $x$ the same coordinates in the primed system as $\bar{x}$ has in the unprimed system, that is $x^{\mu^{\prime}}=\bar{x}^{\mu}$. We can restrict attention to an infinitesimal mapping defined by a (contravariant) vector field $\xi^{\mu}(x)$

$$
\bar{x}^{\mu}=x^{\mu}+\varepsilon \xi^{\mu}
$$

If this mapping is a possible motion of a symmetry group then $\xi^{\mu}(x)$ is referred to as a symmetry generator. The associated transformations of tensors is defined in the following way. Considering first the (covariant) vector field $A_{\mu}(x)$ we define the transformed field $\bar{A}_{\mu}(x)$ so that its components in the primed coordinates 
at the point $x, \bar{A}_{\mu^{\prime}}(x)$, be equal to the components in the unprimed coordinates of $A_{\mu}$ at $\bar{x}$,

$$
\bar{A}_{\mu^{\prime}}(x)=A_{\mu}(\bar{x}) \text {. }
$$

Therefore

$$
\bar{A}_{\mu}(x)=\frac{\partial x^{v^{\prime}}}{\partial x^{\mu}} \bar{A}_{v^{\prime}}(x)=\left(\delta_{\mu}^{v}+\varepsilon \partial_{\mu} \xi^{v}\right)\left(A_{v}(x)+\varepsilon \xi^{\rho} \partial_{\rho} A_{v}\right) .
$$

The Lie derivative of $A_{\mu}$ with respect to $\xi^{\mu}$ is defined to be

$$
\mathbf{L}_{\xi} A_{\mu}=\lim _{\varepsilon \rightarrow 0} \frac{1}{\varepsilon}\left(\bar{A}_{\mu}(x)-A_{\mu}(x)\right) \text {. }
$$

In our case, it takes the value

$$
\mathbf{L}_{\xi} A_{\mu}=\left(\partial_{\mu} \xi^{\rho}\right) A_{\rho}+\xi^{\rho} \partial_{\rho} A_{\mu} .
$$

Similarly for a general tensor we find

$$
\begin{aligned}
\mathbf{L}_{\xi} T_{\mu \nu \ldots}^{\rho \sigma \ldots}= & \left(\partial_{\mu} \xi^{\lambda}\right) T_{\lambda \nu \ldots}^{\rho \sigma \ldots}+\left(\partial_{\nu} \xi^{\lambda}\right) T_{\mu \lambda \ldots}^{\rho \sigma \ldots}+\ldots \\
& -\left(\partial_{\lambda} \xi^{\rho}\right) T_{\mu \nu \ldots}^{\lambda \sigma \ldots}-\left(\partial_{\lambda} \xi^{\sigma}\right) T_{\mu \nu \ldots}^{\rho \lambda \ldots}-\ldots \\
& +\xi^{\lambda} \partial_{\lambda} T_{\mu \nu \ldots}^{\rho \sigma \ldots},
\end{aligned}
$$

which preserves the tensor type. The Lie derivative of a tensor product is given by the Leibnitz rule and contractions are respected, so that the order of Lie derivation of a tensor and contraction of indices is immaterial. The vector field $A_{\mu}$ is said to possess the symmetry generated by $\xi^{\mu}$ if

$$
\bar{A}_{\mu}(x)=A_{\mu}(x),
$$

which implies that the Lie derivative $\mathbf{L}_{\xi} A_{\mu}$ vanishes.

We now generalize these ideas to gauge theories. Suppose that the gauge group $G$ has generators $T^{a}$ with commutation relations $\left[T^{a}, T^{b}\right]=g^{a b c} T^{c}$, and normalization $\operatorname{Tr}\left(T^{a} T^{b}\right)=-2 \delta^{a b}$. Under a gauge transformation $g(x) \in G$, the gauge field, $A_{\mu}(x)=A_{\mu}^{a}(x) T^{a}$, has the transformation rule

$$
A_{\mu}^{g}(x)=g A_{\mu} g^{-1}+\left(\partial_{\mu} g\right) g^{-1} .
$$

For the gauge field, we impose the weakened form of the symmetry requirement (2.8), that for some particular $g_{0}(x)$

$$
\bar{A}_{\mu}(x)=A_{\mu}^{g_{0}}(x) \text {. }
$$

For an infinitesimal mapping, the gauge transformation must be of the infinitesimal form

$$
A_{\mu}^{g_{0}}(x)=A_{\mu}(x)+\varepsilon\left(\partial_{\mu} W-\left[A_{\mu}, W\right]\right) \equiv A_{\mu}(x)+\varepsilon D_{\mu} W,
$$

for some $W=W^{a}(x) T^{a}$ in the Lie algebra of $G$. The symmetry condition (2.10) now implies

$$
\mathbf{L}_{\xi} A_{\mu}=D_{\mu} W
$$

which we call the symmetry equation for $A_{\mu}$. 
If we suppose that $W$ is a coordinate scalar and that it has the gauge transformation rule

$$
\begin{aligned}
W^{g} & =g W g^{-1}+\xi^{\rho}\left(\partial_{\rho} g\right) g^{-1}, \\
& =g W g^{-1}+\left(\mathbf{L}_{\xi} g\right) g^{-1},
\end{aligned}
$$

then the symmetry equation (2.12) is invariant under general coordinate transformations and more importantly, is gauge invariant. Now, given any vector field $\xi^{\mu}(x)$, there is always a choice of coordinates so that $\xi^{\mu^{\prime}}=(1,0, \ldots, 0)$. In these coordinates, the gauge transformation

$$
g=\mathscr{P} \exp -\int_{0}^{x^{1}} W\left(y, x^{2}, \ldots, x^{D}\right) d y,
$$

gauges $W$ away, so that in this gauge and these coordinates the symmetry of $A_{\mu}$ reduces to the statement that $A_{\mu}$ is independent of $x^{1}$. When there are several symmetries, and we cannot simultaneously perform the necessary coordinate and gauge transformations on each symmetry equation, then the general form of the symmetry conditions is essential.

It should be noted that our symmetry conditions apply to only the (coordinate) covariant components of the gauge field. With no assumptions about the metric tensor, the contravariant components do not possess any symmetry. Later, we shall consider the metric in more detail.

We consider now a gauge field which possesses several symmetries

$$
\left(\partial_{\mu} \xi_{m}^{\rho}\right) A_{\rho}+\xi_{m}^{\rho} \partial_{\rho} A_{\mu}=D_{\mu} W_{m}, \quad 1 \leqq m \leqq N .
$$

Clearly, this implies a vector space of symmetries, since if

$$
\xi^{\mu}(x)=\lambda_{m} \xi_{m}^{\mu}(x),
$$

is any linear combination of the $\xi_{m}$ with constant coefficients, then

$$
\mathbf{L}_{\xi} A_{\mu}=D_{\mu}\left(\lambda_{m} W_{m}\right)
$$

We can assume that all the symmetries of $A_{\mu}$ belong to this vector space.

Now, if $\xi_{m}, \xi_{n}$ are two particular symmetry generators, then the commutator of the corresponding Lie derivatives gives

$$
\left(\mathbf{L}_{\xi_{m}} \mathbf{L}_{\xi_{n}}-\mathbf{L}_{\xi_{n}} \mathbf{L}_{\xi_{m}}\right) A_{\mu}=\mathbf{L}_{\xi_{m}}\left(D_{\mu} W_{n}\right)-\mathbf{L}_{\xi_{n}}\left(D_{\mu} W_{m}\right)
$$

This reduces to

$$
\mathbf{L}_{\eta} A_{\mu}=D_{\mu}\left(\mathbf{L}_{\xi_{m}} W_{n}-\mathbf{L}_{\xi_{n}} W_{m}-\left[W_{m}, W_{n}\right]\right),
$$

where $\eta$ is the Lie bracket of $\xi_{m}$ and $\xi_{n}$, defined by

$$
\eta^{\mu}=\left[\xi_{m}, \xi_{n}\right]^{\mu} \equiv \xi_{m}^{\rho} \partial_{\rho} \xi_{n}^{\mu}-\xi_{n}^{\rho} \partial_{\rho} \xi_{m}^{\mu}
$$

This new symmetry equation must belong to the vector space, so that for some constants $f_{m n p}$ we must have

$$
\left[\xi_{m}, \xi_{n}\right]^{\mu}=f_{m n p} \xi_{p}^{\mu}
$$


and then, for consistency, we require

$$
\mathbf{L}_{\xi_{m}} W_{n}-\mathbf{L}_{\xi_{n}} W_{m}-\left[W_{m}, W_{n}\right]-f_{m n p} W_{p}=0 .
$$

The Lie bracket is antisymmetric and satisfies the Jacobi identity, so that closure under the Lie bracket of a vector space of vector fields is a prototype Lie algebra. The structure constants $f_{m n p}$ satisfy the corresponding properties for an abstract Lie algebra

$$
\begin{aligned}
& f_{m n p}=-f_{n m p}, \\
& f_{m n p} f_{p q r}+f_{n q p} f_{p m r}+f_{q m p} f_{p n r}=0 .
\end{aligned}
$$

In fact, to obtain eq. (2.22) we have assumed that there is no $\psi$ for which $D_{\mu} \psi$ vanishes. For if

$$
\partial_{\mu} \psi-\left[A_{\mu}, \psi\right]=0,
$$

we can gauge transform $\psi$ into a Cartan subalgebra of the gauge group, and then $\psi$ must be constant and $A_{\mu}$ commute with $\psi$. The theory reduces to one whose gauge group is the little group of $\psi$, and this should be considered in its own right.

The definition of spherical symmetry used by previous authors is a particular case of our formalism. The three symmetry generators of 3-D rotations in Cartesian coordinates $\xi_{m}^{i}=\varepsilon^{m i j} x^{j}$ satisfy the $\mathrm{SO}(3)$ algebra

$$
\left[\xi_{m}, \xi_{n}\right]^{i}=\varepsilon_{m n p} \xi_{p}^{i}
$$

and the consistency equation (2.22) has a constant solution $W_{m}=-\mathscr{T}^{m}$, where $\mathscr{T}^{m}$ generate an $\mathrm{SO}(3)$ subgroup of the gauge group. The symmetry equation then takes the form

$$
\varepsilon_{i j k} x_{j} \partial_{k} A_{l}+\varepsilon_{i l k} A_{k}-\left[\mathscr{T}^{i}, A_{l}\right]=0,
$$

where we recognize the terms of the combined rotation $\mathscr{J}_{i}$, in (1.1).

Returning to the general case, we can derive, from the symmetry of the gauge field, the corresponding symmetry of the field tensor $F_{\mu \nu}=\partial_{\mu} A_{\nu}-\partial_{\nu} A_{\mu}-\left[A_{\mu}, A_{\nu}\right]$. Equation (2.12) implies that

$$
\begin{aligned}
D_{v} \mathbf{L}_{\xi} A_{\mu}-D_{\mu} \mathbf{L}_{\xi} A_{v} & =\left(D_{v} D_{\mu}-D_{\mu} D_{v}\right) W, \\
& =-\left[F_{v \mu}, W\right] .
\end{aligned}
$$

This simplifies to

$$
\mathbf{L}_{\xi} F_{v \mu}=-\left[F_{v \mu}, W\right]
$$

which is the symmetry condition we expect for a gauge covariant tensor.

We conclude this section with a discussion of certain scalar fields with interesting and useful symmetry properties. It was pointed out by Lohe [11], that if a gauge field is time-independent, then the time component $A_{0}$ could be interpreted as a scalar Higgs field $\phi$. Part of the field tensor then reduces to a covariant derivative $F_{0 i}=-D_{i} \phi$. Olive has obtained striking results in the quantum theory of monopoles by developing this idea [12]. We can obtain a generalization of Lohe's 
result. Using the symmetry equations (2.15), we find

$$
\begin{aligned}
\xi_{m}^{\mu} F_{\mu \nu} & =\xi_{m}^{\mu}\left(\partial_{\mu} A_{v}-\partial_{v} A_{\mu}-\left[A_{\mu}, A_{v}\right]\right), \\
& =D_{v} W_{m}-\left(\partial_{v} \xi_{m}^{\mu}\right) A_{\mu}-\xi_{m}^{\mu} \partial_{v} A_{\mu}-\left[\xi_{m}^{\mu} A_{\mu}, A_{v}\right], \\
& =D_{v}\left(W_{m}-\xi_{m}^{\mu} A_{\mu}\right) .
\end{aligned}
$$

Defining the scalar field $\Psi_{m}=\xi_{m}^{\mu} A_{\mu}-W_{m}$, we have reduced part of the field tensor to a covariant derivative

$$
\xi_{m}^{\mu} F_{\mu \nu}=-D_{\nu} \Psi_{m},
$$

a result which is independent of the gauge. The fields $\Psi_{m}$ are related to, but not the same as, the Higgs fields we introduce later. They possess the symmetry property (no summation over $m$ )

$$
\mathbf{L}_{\xi_{m}} \Psi_{m}-\left[W_{m}, \Psi_{m}\right]=0,
$$

which we expect for a covariant scalar field, and from (2.9) and (2.13) we see that $\Psi_{m}$ is indeed covariant. We can obtain a stronger result than (2.32) if we use the symmetry equations for $A_{\mu}$, together with the consistency conditions (2.21), (2.22). We find:

$$
\mathbf{L}_{\xi_{m}} \Psi_{n}-\left[W_{m}, \Psi_{n}\right]=f_{m n p} \Psi_{p}
$$

from which we can now calculate a double contraction of the field tensor

$$
\begin{aligned}
\xi_{m}^{\mu} \xi_{n}^{v} F_{\mu \nu} & =-\xi_{n}^{v}\left(\partial_{v} \Psi_{m}-\left[A_{v}, \Psi_{m}\right]\right), \\
& =-\mathbf{L}_{\xi_{n}} \Psi_{m}+\left[W_{n}+\Psi_{n}, \Psi_{m}\right], \\
& =f_{m n p} \Psi_{p}-\left[\Psi_{m}, \Psi_{n}\right] .
\end{aligned}
$$

We see that the field tensor has certain components which are expressible as covariant derivatives of $\Psi_{m}$ and some which are algebraic and quadratic in $\Psi_{m}$. This will allow us later to reduce the action to that of a Higgs model with a quartic potential.

\section{Construction of the Symmetry Generators}

In this section, we show how to find all symmetry generators satisfying a given Lie bracket algebra, then consider the consistency equation (2.22) for the fields $W_{m}$, and finally combine the results to simplify the symmetry equations for the gauge field. First, we calculate solutions of the equation

$$
\left[\xi_{m}, \xi_{n}\right]^{\mu}=f_{m n p} \xi_{p}^{\mu}
$$

Suppose that, at any given point, the vectors $\xi_{m}$ span an $N^{\prime}$-dimensional subspace of the complete $D$-dimensional (tangent) space. Then, by Frobenius's theorem, there exists a family of $N^{\prime}$-dimensional surfaces, $\mathscr{X}$, to which $\xi_{m}$ are tangent, if Eq. (3.1) is satisfied. We shall restrict our attention to a single coordinate neighbourhood, and ignore global problems throughout. Then we can choose coordinates for the manifold $\mathscr{M}$

$$
x^{\mu}=\left(x^{i}, y^{\alpha}\right), \quad 1 \leqq i \leqq D^{\prime}, 1 \leqq \alpha \leqq N^{\prime}, N^{\prime}+D^{\prime}=D,
$$


so that these surfaces are defined by $x^{i}=$ constant, and the components of $\xi_{m}$ are then $\xi_{m}^{\mu}=\left(0, \xi_{m}^{\alpha}\right)$. Because the derivatives we shall consider are directed along $\xi_{m}$, we may now work on a given surface, $\mathscr{X}_{0}$, with $x^{i}$ constant, so that for the remainder of this section we regard $\xi_{m}, W_{m}$ as functions of $y$ but not of $x$, and we treat $\xi_{m}$ as the $N^{\prime}$-dimensional vector with components $\xi_{m}^{\alpha}$. We also temporarily ignore the components $A_{i}\left(1 \leqq i \leqq D^{\prime}\right)$ of the gauge field.

The fundamental solution of Eq. (3.1) is the set of infinitesimal right translations on the symmetry group, $S . S$ is the abstract Lie group with constant matrix genetors $J^{m}$, and the same structure constants $f_{m n p}$, as in the Lie bracket algebra (3.1), so

$$
\left[J^{m}, J^{n}\right]=f_{m n p} J^{p} .
$$

We now assume this algebra is semi-simple, so that the structure constants are totally antisymmetric, and normalized by $f_{m n p} f_{m n r}=2 \delta_{p r}$. For our purposes, the group is unique since we are working on a coordinate neighbourhood.

Let $s \in S$ have coordinates $y^{\hat{\alpha}}(e . g$. Euler angles for $\operatorname{SO}(3))$. Then,

$$
s\left(1+\varepsilon J^{m}\right)=s+\varepsilon \xi_{m}^{\hat{\alpha}} \partial_{\hat{\alpha}} s,
$$

where $y^{\hat{\alpha}}+\varepsilon \xi_{m}^{\hat{\alpha}}$ are the coordinates of $s\left(1+\varepsilon J^{m}\right)$, thus defining the infinitesimal right translations $\xi_{m}^{\hat{\alpha}}(y)$ on the group. We can write (3.4) as

$$
s J^{m}=\mathbf{L}_{\xi_{m}} s .
$$

Since $J^{m}$ is constant

$$
\mathbf{L}_{\xi_{m}} \mathbf{L}_{\xi_{n}} s=s J^{m} J^{n}
$$

from which we derive, for all $y^{\hat{\alpha}}$,

$$
\mathbf{L}_{\left[\xi_{m}, \xi_{n}\right]} s=f_{m n p} \mathbf{L}_{\xi_{p}} s,
$$

so that (3.1) is satisfied. For a Lie group of dimension $N, \xi_{m}^{\hat{\alpha}}(y)$ is an $N \times N$ matrix which is non-singular everywhere.

We obtain further solutions of (3.1) as sets of right translations on right coset spaces of $S$. Let $R$ be a Lie subgroup of $S$, of dimension $N-N^{\prime}$, with generators $J^{m},\left(N^{\prime}+1 \leqq m \leqq N\right)$, and let the coset $R s$ have coordinates $y^{\alpha}$. As before

$$
R s\left(1+\varepsilon J^{m}\right)=R s+\varepsilon \xi_{m}^{\alpha} \partial_{\alpha}(R s),
$$

where $y^{\alpha}+\varepsilon \xi_{m}^{\alpha}$ are the coordinates of the coset $R s\left(1+\varepsilon J^{m}\right)$. It is easy to show again that $\xi_{m}^{\alpha}(y)$ satisfy (3.1).

In fact these are the only solutions of (3.1), for the Lie group $S$ always acts as a transformation group on the surface $\mathscr{X}_{0}$, that is, as a group of mappings, $S \times \mathscr{X}_{0} \rightarrow \mathscr{X}_{0}$. Suppose $R$ is the subgroup of $S$ which maps a given point $z_{0}$ onto itself. Let $z$ be any point and suppose $s \in S$ maps $z$ to $z_{0}$. Then all elements of the coset $R s$ map $z$ to $z_{0}$, and we may.identify $z$ with $R s$. We can therefore always identify the surface $\mathscr{X}_{0}$ with some coset space $S / R$ and $\xi_{m}$ with the corresponding infinitesimal right translations.

At this point, we clarify our use of coordinates. We have used $y^{\alpha}$ (early greek indices) interchangeably for $\mathscr{X}_{0}$ and the coset space $S / R$, and use $y^{\omega}$ (late greek 
indices) as coordinates of the subgroup $R$. If we fix an origin

$$
s_{0}\left(y^{\alpha}\right) \in R s\left(y^{\alpha}\right),
$$

for each coset in a smooth way, then every element of $S$ can be written uniquely, with coordinates $y^{\hat{\alpha}}=\left(y^{\omega}, y^{\alpha}\right)$, as

$$
s\left(y^{\hat{\alpha}}\right)=r\left(y^{\omega}\right) s_{0}\left(y^{\alpha}\right),
$$

for some $r \in R$. Writing

$$
\xi_{m}^{\hat{\alpha}}=\left(\xi_{m}^{\omega}, * \xi_{m}^{\alpha}\right)
$$

on the group $S$, it is easy to see that the components $* \xi_{m}^{\alpha}$, which are independent of $y^{\omega}$, are in fact the symmetry generators on the coset space, denoted before by $\xi_{m}^{\alpha}$, that is

$$
* \xi_{m}^{\alpha}=\xi_{m}^{\alpha} \text {. }
$$

These coset space generators are therefore obtained from the symmetry generators on the group by projection.

We can now proceed to the consistency equation for the fields $W_{m}$, Eq. (2.22). In a sense to be explained, the solution is always $W_{m}=0$. Assume that $\xi_{m}^{\alpha}$ are the right translations on $S / R$. The $W_{m}$ are defined on the coset space, but now we embed our coset space problem into the whole group $S$. We regard $W_{m}$ formally as defined on the group, but constant on any coset, so that

$$
W_{m}\left(y^{\omega}, y^{\alpha}\right)=W_{m}\left(y^{\alpha}\right) \quad \forall y^{\alpha} .
$$

Since $\xi_{m}^{\alpha}$ is a projection of $\xi_{m}^{\hat{\alpha}}$, and $W_{m}$ is independent of $y^{\omega}$, we can identify the corresponding Lie derivatives of $W_{m}$

$$
\xi_{n}^{\hat{\alpha}} \partial_{\hat{\alpha}} W_{m}=\xi_{n}^{\alpha} \partial_{\alpha} W_{m}
$$

so that $W_{m}$ is a solution of the consistency equation on $S$

$$
\xi_{m}^{\hat{\alpha}} \partial_{\hat{\alpha}} W_{n}-\xi_{n}^{\hat{\alpha}} \partial_{\hat{\alpha}} W_{m}-\left[W_{m}, W_{n}\right]-f_{m n p} W_{p}=0,
$$

with the restriction that $W_{m}$ is independent of $y^{\omega}$.

On the group, $\xi_{m}^{\hat{\alpha}}$ form an invertible matrix, so we can unambiguously define new fields $W_{\hat{\alpha}}$, which in general are not constant on each coset, by

$$
W_{m}=\xi_{m}^{\hat{\alpha}} W_{\hat{\alpha}} \text {. }
$$

In terms of $W_{\hat{\alpha}}$, Eq. (3.15) simplifies to

$$
\partial_{\hat{\alpha}} W_{\hat{\beta}}-\partial_{\hat{\beta}} W_{\hat{\alpha}}-\left[W_{\hat{\alpha}}, W_{\hat{\beta}}\right]=0 \text {, }
$$

so that $W_{\hat{\alpha}}$ is a pure gauge defined on the symmetry group,

$$
W_{\hat{\alpha}}=\left(\partial_{\hat{\alpha}} g\right) g^{-1} \text {. }
$$

By embedding our problem in the symmetry group, we have enlarged the class of gauge transformations to include those that depend on $y^{\omega}$, and from (2.13) we see that $W_{\hat{\alpha}}$ transforms as an ordinary gauge field on $S$. By a choice of gauge we can simply set $W_{\hat{\alpha}}=0$ and hence $W_{m}=0$ on the symmetry group. To obtain 
solutions on the coset space, we would require $W_{m}$, given by

$$
W_{m}=\xi_{m}^{\hat{\alpha}}\left(\partial_{\hat{\alpha}} g\right) g^{-1},
$$

to be independent of $y^{\omega}$. In general, such a $W_{m}$ cannot be gauge transformed to zero on the coset space because the gauge transformations cannot depend on $y^{\omega}$. In explicit examples, we have been able to calculate these $W_{m}$ and then find gauge fields satisfying the symmetry equations.

We have developed more powerful tools, however, realizing that the symmetry equation itself can be embedded into the whole symmetry group. Recall that on the surface $\mathscr{X}_{0}$, identified with the coset space $S / R$, we are considering just the components of the gauge field $A_{\alpha}\left(y^{\beta}\right)$. But now, as before, we regard $A_{\alpha}$ formally as defined on the symmetry group but constant on any coset, and we introduce extra components $A_{\omega}=0$ corresponding to the subgroup coordinates $y^{\omega}$, so

$$
A_{\hat{\alpha}}\left(y^{\hat{\beta}}\right) \equiv\left(A_{\omega}\left(y^{\hat{\beta}}\right), A_{\alpha}\left(y^{\hat{\beta}}\right)\right)=\left(0, A_{\alpha}\left(y^{\beta}\right)\right) \text {. }
$$

Then, any solution of the symmetry equation on the coset space is a special case of a solution on the group. To see this, we split the equation on the group as follows

$$
\begin{aligned}
& \left(\partial_{\omega} \xi_{m}^{\hat{\alpha}}\right) A_{\hat{\alpha}}+\xi_{m}^{\hat{\alpha}} \partial_{\hat{\alpha}} A_{\omega}=\partial_{\omega} W_{m}-\left[A_{\omega}, W_{m}\right], \\
& \left(\partial_{\beta} \xi_{m}^{\hat{\alpha}}\right) A_{\hat{\alpha}}+\xi_{m}^{\hat{\alpha}} \partial_{\hat{\alpha}} A_{\beta}=\partial_{\beta} W_{m}-\left[A_{\beta}, W_{m}\right] .
\end{aligned}
$$

The first of these equations is automatically satisfied, remembering that $W_{m}, \xi_{m}^{\alpha}$ are independent of $y^{\omega}$. The second reduces to the symmetry equation on the coset space.

It is not difficult to solve the symmetry equation on $S$. We can use the enlarged gauge freedom to set $W_{m}=0$, and the symmetry equation reduces to

$$
\mathbf{L}_{\xi_{m}} A_{\hat{\alpha}}=0 \text {. }
$$

Written in this way the gauge group decouples and we can solve for each component separately:

$$
\mathbf{L}_{\xi_{m}} A_{\hat{\alpha}}^{a}=0 \text {. }
$$

This equation is well known in Lie group theory, and will be discussed in the next section.

\section{Solution of the Symmetry Equations}

An invariant tensor on the Lie group $S$ satisfies

$$
\mathbf{L}_{\xi_{m}} T_{\hat{\alpha} \hat{\beta} \ldots .}^{\hat{\gamma} \hat{\delta} \ldots=0,}
$$

for all infinitesimal right translations $\xi_{m}$. Our aim is to find vectors which are invariant, to solve (3.23), but for later purposes our discussion is more general. We can define infinitesimal left translations $\tilde{\xi}_{m}^{\hat{\alpha}}$, so that

$$
\mathbf{L}_{\xi_{m}} s=s J^{m}, \quad \mathbf{L}_{\tilde{\xi}_{m}} s=-J^{m} s .
$$

The vector fields $\tilde{\xi}_{m}$ satisfy the same Lie bracket algebra as the $\xi_{m}$, and are related 
to $\xi_{m}$ by the coordinate transformation which maps each group element to its inverse. At the identity, we see that $\xi_{m}=-\widetilde{\xi}_{m}$.

The commutator of $\mathbf{L}_{\xi_{m}}$ and $\mathbf{L}_{\widetilde{\xi}_{n}}$ vanishes, since

$$
\mathbf{L}_{\widetilde{\xi}_{n}} \mathbf{L}_{\xi_{m}} s=-J^{n} S J^{m}=\mathbf{L}_{\xi_{m}} \mathbf{L}_{\widetilde{\xi}_{n}} s
$$

and therefore

$$
\left[\xi_{m}, \tilde{\xi}_{n}\right]=0, \quad \forall m, n
$$

But the Lie bracket is identical to the Lie derivative in this case, so

$$
\mathbf{L}_{\xi_{m}} \tilde{\xi}_{n}^{\hat{\alpha}}=0, \quad \forall m, n
$$

and therefore, $\tilde{\xi}_{m}^{\hat{\alpha}}$ are invariant vector fields.

We can define covariant vector fields $\widetilde{\xi}_{m \hat{\alpha}}$ by inverting the matrix $\tilde{\xi}_{m}^{\hat{\alpha}}$, as follows

$$
\xi_{m \hat{\alpha}} \tilde{\xi}_{n}^{\hat{\alpha}}=\delta_{m n},
$$

which immediately implies another orthogonality relation

$$
\tilde{\xi}_{m \hat{\alpha}} \tilde{\xi}_{m}^{\hat{\beta}}=\delta_{\hat{\alpha}}^{\hat{\beta}}
$$

Fields $\xi_{m \hat{\alpha}}$ are similarly defined so that

$$
\xi_{m \hat{\alpha}} \xi_{n}^{\hat{\alpha}}=\delta_{m n}, \quad \xi_{m \hat{\alpha}} \xi_{m}^{\hat{\beta}}=\delta_{\hat{\alpha}}^{\hat{\beta}}
$$

Since $\delta_{m n}$ is a constant scalar, all of whose Lie derivatives vanish, we can use the Leibnitz rule to show that

$$
\mathbf{L}_{\xi_{m}} \tilde{\xi}_{n \hat{\alpha}}=0, \quad \forall m, n \text {. }
$$

Finally we can write down a general invariant tensor

$$
T_{\hat{\alpha} \hat{\beta} \ldots}^{\hat{\gamma} \hat{\delta} \ldots}=\lambda_{m n \ldots p q \ldots} \xi_{m \hat{\alpha}} \xi_{n \hat{\beta} \ldots} \xi_{p}^{\hat{\gamma}} \tilde{\xi} \hat{\delta} \ldots
$$

where $\lambda_{m n \ldots p q \ldots}$ is a set of constants. All Lie derivatives $\mathbf{L}_{\xi_{m}} T$ vanish, by the Leibnitz rule. Indeed, these are the only invariant tensors, for the vectors $\widetilde{\xi}_{m}^{\hat{\alpha}}$ form a spanning set at any point, as do the vectors $\widetilde{\xi}_{m \hat{\alpha}}$. The most general tensor can then be written in the form of (4.10) with $\lambda$ position dependent. The Leibnitz rule shows that for $T$ to be invariant, $\lambda$ must however be constant.

Of special interest is the invariant tensor

$$
\tilde{h}^{\hat{\alpha} \hat{\beta}}=\widetilde{\xi}_{m}^{\hat{\alpha}} \widetilde{\xi}_{m}^{\hat{\beta}} \text {. }
$$

This acts as a raising operator, since from Eq. (4.6)

$$
\tilde{\xi}_{m \hat{\alpha}} \tilde{h}^{\hat{\alpha} \hat{\beta}}=\tilde{\xi}_{m}^{\hat{\beta}}
$$

so that $\tilde{h}^{\hat{\alpha} \hat{\beta}}$ is the (left) metric tensor on the Lie group. The related (right) metric tensor $h^{\hat{\alpha} \hat{\beta}}=\xi_{m}^{\hat{\alpha}} \xi_{m}^{\hat{\beta}}$ is also invariant, since

$$
\begin{aligned}
& \mathbf{L}_{\xi_{m}} h^{\hat{\alpha} \hat{\beta}}=\left(\mathbf{L}_{\xi_{m}} \xi_{n}\right)^{\hat{\alpha}} \xi_{n}^{\hat{\beta}}+\xi_{n}^{\hat{\alpha}}\left(\mathbf{L}_{\xi_{m}} \xi_{n}\right)^{\hat{\beta}}, \\
& =f_{m n p} \xi_{p}^{\hat{\alpha}} \xi_{n}^{\hat{\beta}}+f_{m n p} \xi_{n}^{\hat{\alpha}} \xi_{p}^{\hat{\beta}} \text {, } \\
& =0 \text {, }
\end{aligned}
$$


using the total antisymmetry of $f_{m n p}$. Since $h^{\hat{\alpha} \hat{\beta}}$ and $\tilde{h}^{\hat{\alpha} \hat{\beta}}$ are equal at the identity, we conclude that they are equal everywhere, so there is a unique metric tensor. By a similar argument we have the useful equality

$$
f_{m n} \tilde{\xi}_{m}^{\hat{\alpha}} \tilde{\xi}_{n}^{\hat{\beta}} \tilde{\xi}_{p}^{\hat{\gamma}}=-f_{m n p} \xi_{m}^{\hat{\alpha}} \xi_{n}^{\hat{\beta}} \xi_{p}^{\hat{\nu}} \text {. }
$$

The tensor on the 1.h.s. is clearly invariant, and the invariance of the r.h.s. follows from the Jacobi identity for the structure constants. This tensor also has a geometrical significance on the Lie group, which we now show.

The symmetric Riemann-Christoffel connection, obtained from the group metric, is

$$
\begin{aligned}
\left\{\begin{array}{c}
\hat{\gamma} \\
\hat{\beta} \hat{\alpha}
\end{array}\right\} & =\frac{1}{2} h^{\hat{\hat{\gamma}}}\left(\partial_{\hat{\beta}} h_{\hat{\alpha} \hat{\hat{\varepsilon}}}+\partial_{\hat{\alpha}} h_{\hat{\beta} \hat{\varepsilon}}-\partial_{\hat{\varepsilon}} h_{\hat{\beta} \hat{\alpha}}\right), \\
& =-\xi_{m \hat{\beta}} \partial_{\hat{\alpha}} \xi_{m}^{\hat{\gamma}}+\frac{1}{2} f_{m n p} \xi_{m}^{\hat{\gamma}} \xi_{n \hat{\alpha}} \xi_{p \hat{\beta}},
\end{aligned}
$$

but it is more natural to define parallel transport of tensors by the requirement that they be invariant in the Lie derivative sense. In that case, the connection is

$$
\Gamma_{\hat{\alpha} \hat{\beta}}^{\hat{\gamma}}=-\xi_{m \hat{\beta}} \partial_{\hat{\alpha}} \hat{\zeta}_{m}^{\hat{\gamma}},
$$

which is not symmetric, and the invariance of tensors in reinterpreted as the vanishing of (coordinate) covariant derivatives. The tensor appearing in Eq. (4.14) is then the torsion, the antisymmetric part of this connection. We note that with the symmetric connection, the invariant curvature tensor is

$$
R_{\hat{\beta} \hat{\gamma} \hat{\delta}}^{\hat{\alpha}}=\frac{1}{4} f_{m n p} f_{m q r} \xi_{n}^{\hat{\alpha}} \xi_{p \hat{\beta}} \xi_{q q \hat{\gamma}} \xi_{r \hat{\delta}},
$$

and the torsion is zero, whereas the connection (4.16) with torsion has vanishing curvature.

Now we consider the symmetry equation, which in the gauge where $W_{m}=0$ is

$$
L_{\xi m} A_{\hat{\alpha}}^{a}=0, \quad \forall m .
$$

We have seen that the solution of this equation is

$$
A_{\hat{\alpha}}^{a}=\Phi_{m}^{a} \tilde{\xi}_{m \hat{\alpha}},
$$

where the coefficients $\Phi_{m}^{a}$ are independent of $y^{\hat{\alpha}}$. We now reintroduce the coordinates $x^{i}$, which were suppressed earlier, and the corresponding components of the gauge fièld. Still embedding the coset space into the symmetry group, and in the gauge where $W_{m}=0$, the complete solution of the symmetry equation can be expressed, in terms of arbitrary fields $A_{i}^{a}(x)$ and $\Phi_{m}^{a}(x)$ defined on the space $\mathscr{X}$, as

$$
\begin{aligned}
& A_{i}^{a}=A_{i}^{a}(x), \\
& A_{\hat{\alpha}}^{a}=\Phi_{m}^{a}(x) \tilde{\xi}_{m \hat{\alpha}}(y) .
\end{aligned}
$$

The scalar fields $\Psi_{m}$ are given by

$$
\Psi_{m}=\xi_{m}^{\hat{\alpha}} \Phi_{n} \xi_{n \hat{\alpha}},
$$

where $\Phi_{n}=\Phi_{n}^{a} T^{a}$. We know that under a gauge transformation, $\Psi_{m}$ transforms covariantly. This will be realized if we define $\Phi_{m}$ to transform covariantly as well, 
so that Eq. (4.22) is gauge invariant. The fields $\Phi_{m}$ appear as Higgs fields in the dimensionally reduced Lagrangian, later.

The solution (4.19) for $A_{\hat{\alpha}}$ is the most general one on the symmetry group, but we must now impose constraints on $\Phi_{m}$ and $A_{i}$ to ensure that we obtain a solution on the coset space. We require, in some gauge, that the components $A_{\omega}$ vanish and that the non-vanishing components be independent of $y^{\omega}$. A sufficient, and obviously necessary, condition for this is that all the components of the field tensor $F_{i \omega}, F_{\alpha \omega}, F_{\tau \omega}$ vanish, which is a gauge invariant condition. For if all components $F_{\tau \omega}$, corresponding to the subgroup coordinates, vanish, then there is a gauge where $A_{\omega}=0$, and in such a gauge $F_{i \omega}, F_{\alpha \omega}$ are zero only if $A_{i}, A_{\alpha}$ are independent of $y^{\omega}$.

To calculate the field tensor, we use the expressions (2.31), (2.34), or rather the obvious extension of these results to the symmetry group

$$
\begin{aligned}
& \xi_{m}^{\hat{\alpha}} F_{\hat{\alpha} i}=-D_{i} \Psi_{m}, \\
& \xi_{m}^{\hat{\alpha}} \xi_{n}^{\hat{\beta}} F_{\hat{\alpha} \hat{\beta}}=f_{m n p} \Psi_{p}-\left[\Psi_{m}, \Psi_{n}\right] .
\end{aligned}
$$

Inserting the form (4.22) for $\Psi_{m}$, and using the property of the torsion (4.14) as well as the orthogonality properties of $\tilde{\xi}_{m}$ we obtain

$$
\begin{aligned}
& F_{\hat{\alpha} i}=\tilde{\xi}_{m \hat{\alpha}}\left(\partial_{i} \Phi_{m}-\left[A_{i}, \Phi_{m}\right]\right), \\
& F_{\hat{\alpha} \hat{\beta}}=-\xi_{m \hat{\alpha}} \tilde{\xi}_{n \hat{\beta}}\left(f_{m n p} \Phi_{p}+\left[\Phi_{m}, \Phi_{n}\right]\right) .
\end{aligned}
$$

Thus, for $F_{i \omega}, F_{\hat{\alpha} \omega}$ to vanish in this gauge, and hence in any gauge, we require

$$
\begin{aligned}
& \tilde{\xi}_{n \omega}\left(\partial_{i} \Phi_{n}-\left[A_{i}, \Phi_{n}\right]\right)=0, \\
& \tilde{\xi}_{n \omega}\left(f_{m n p} \Phi_{p}+\left[\Phi_{m}, \Phi_{n}\right]\right)=0 .
\end{aligned}
$$

It is easy to see what a condition of the form $\lambda_{n} \xi_{n \omega}=0$ means, since (3.10) and (4.2) imply that

$$
\left(\partial_{\omega} r\right) r^{-1}=-J^{m} \tilde{\xi}_{m \omega} .
$$

For $m>N^{\prime}$ (that is, $J^{m}$ a generator of $R$ ), $\tilde{\xi}_{m \omega}$ are therefore the infinitesimal left translations on the subgroup $R$ and for $m \leqq N^{\prime}, \tilde{\xi}_{m \omega}$ are zero. For $\lambda_{n} \tilde{\xi}_{n \omega}$ to vanish, therefore, we require $\lambda_{n}=0$ for $n>N^{\prime}$ so the equations (4.27), (4.28) reduce to the constraints

$$
\begin{aligned}
& \partial_{i} \Phi_{n}-\left[A_{i}, \Phi_{n}\right]=0, \quad \forall i, \forall n>N^{\prime}, \\
& f_{m n p} \Phi_{p}+\left[\Phi_{m}, \Phi_{n}\right]=0, \quad \forall m, \forall n>N^{\prime} .
\end{aligned}
$$

We see from Eq. (4.31) that $\Phi^{\prime}=\left\{-\Phi_{m}: m>N^{\prime}\right\}$ generate an $R$ subalgebra of the gauge group algebra, though not necessarily faithfully. We shall assume from now on, however, that $R$ is a subgroup of $G$ generated faithfully by $\Phi^{\prime}$. Now, we wish to obtain a dimensionally reduced action involving completely unconstrained fields. Although the embedding of $R$ into $G$ could vary continuously with $x$, there is no way that any element of $\Phi^{\prime}$ could be completely arbitrary. Also, because of the constraint (4.30), these fields would have no kinetic term. We therefore fix the elements of $\Phi^{\prime}$ to be constant, which is possible by a $y$-independent 
gauge transformation leaving the form of the gauge fields (4.20), (4.21) unchanged.

The first constraint now requires that $A_{i}$ commute with all elements of $\Phi^{\prime}$, that is, $A_{i}$ are gauge fields for the little group of $\Phi^{\prime}$. This little group is the residual gauge group of the action after dimensional reduction. This follows from the observation that solutions of both constraint equations are generally gauge invariant, but only gauge transformations in the little group keep the elements of $\Phi^{\prime}$ fixed. We have analysed the second constraint (4.31), but since we have not explicitly used the results, the details can be found in the Appendix. This constraint equation relates the induced representations, denoted by $M^{\prime}$ and $M^{\prime \prime}$ respectively, of $R$ over $S / R$ and of $R$ over $G$. If we denote by $M_{i}^{\prime}$ and $M_{i}^{\prime \prime}$ the irreducible components of $M^{\prime}$ and $M^{\prime \prime}$, then corresponding to each pair $\left(M_{i}^{\prime}, M_{j}^{\prime \prime}\right)$ where the two components are the same, there is a single non-vanishing, and arbitrary, residual Higgs field component.

The constraints guarantee that the gauge fields on $S$ can be gauge transformed to a solution of the symmetry equations on the original space. We have identified $R$ as both a subgroup of $S$ and of $G$, by identifying, for $m>N^{\prime}$, the generators $J^{m}$ and $-\Phi_{m}=-\Phi_{m}^{a} T^{a}$. Since on the symmetry group

$$
A_{\omega}=\Phi_{m} \widetilde{\xi}_{m \omega},
$$

we deduce from (4.29) that $A_{\omega}$ is the pure gauge generated by $r\left(y^{\omega}\right)$. The gauge transformation that makes $A_{\omega}$ vanish, and the other components independent of $y^{\omega}$ is therefore $r^{-1}\left(y^{\omega}\right)$. This gauge transformation leaves $A_{i}$ unchanged, as $A_{i}$ commutes with all the generators $\Phi_{m}\left(m>N^{\prime}\right)$, and transforms $A_{\hat{\alpha}}$ homogeneously, so that the final solution of the symmetry equations on the original manifold $\mathscr{M}$ is

$$
\begin{aligned}
& A_{i}=A_{i}(x), \\
& A_{\alpha}=r^{-1} \Phi_{m} r \tilde{\xi}_{m \alpha} .
\end{aligned}
$$

In this gauge, the components of $W_{\hat{\alpha}}$ are

$$
\begin{aligned}
& W_{\omega}=-r^{-1} \partial_{\omega} r=-r^{-1} \Phi_{m} r \xi_{m \omega}, \\
& W_{\alpha}=0,
\end{aligned}
$$

and the fields $W_{m}=\xi_{m}^{\hat{\alpha}} W_{\hat{\alpha}}$ on $\mathscr{M}$ are

$$
W_{m}=-r^{-1} \Phi_{n} r \xi_{m}^{\omega} \xi_{n \omega}
$$

These fields clearly play the role of the absent gauge field components $A_{\omega}$.

Since, by construction, the fields $A_{\alpha}, W_{m}$ are independent of the subgroup coordinates $y^{\tau}$, they can be calculated for those values, say $y^{\tau}=y_{0}^{\tau}$, for which $r=1$, thereby obtaining the simpler expressions

$$
A_{\alpha}=\left.\Phi_{m} \tilde{\xi}_{m \alpha}\right|_{y^{\tau}=y_{0}^{\tau}}
$$

and,

$$
W_{m}=-\left.\Phi_{n} \xi_{m}^{\omega} \xi_{n \omega}\right|_{y^{\tau}=y_{0}^{\tau}}
$$




\section{Dimensional Reduction of the Action}

We show how the Lagrangian defined on the original manifold $\mathscr{M}$ can be reduced to a Higgs model on the space $\mathscr{X}$. As was already mentioned, we assumed nothing about the metric of $\mathscr{M}$ and can say nothing about the symmetry of the contravariant components of the gauge fields $A^{\mu}(\mu$ is raised by the metric tensor of $\mathscr{M}$ ). With no assumptions about the metric $h^{\mu \nu}\left(x^{i}, y^{\alpha}\right)$ (even if $\mathscr{M}$ is flat) we can say little about the Lagrangian.

This may indeed be the physical situation and we are familiar with one example where this is the case. In a calculation of the force between monopoles [13], it was necessary to assume that the monopoles accelerated rigidly. It can be shown that rigid acceleration of an extended soliton is equivalent to the vanishing of the Lie derivative $\mathbf{L}_{\xi}$ of all fields. In this case $\xi^{\mu}$ generates non-uniform motion and is clearly not a symmetry of the metric of flat space.

We can reduce the Lagrangian only if we make a special ansatz for the metric, so that it is symmetric. A natural ansatz for $h^{\mu v}\left(x^{i}, y^{\alpha}\right)$ is

$$
h^{\mu \nu}=\left(\begin{array}{c|c}
h^{i j}(x) & 0 \\
\hline 0 & \frac{1}{R^{2}(x)} h^{\alpha \beta}(y)
\end{array}\right),
$$

where $h^{i j}(x)$ and $R(x)$ are arbitrary functions and $h^{\alpha \beta}=\xi_{m}^{\alpha} \xi_{m}^{\beta}$ is the projection of the group metric to the coset space, which is invariant on the coset space.

We would like to express the Lagrangian in terms of $\Phi_{m}$ and $A_{i}$ assuming that these are the constrained fields. The original action

$$
\mathscr{L}=-\frac{1}{8} \operatorname{Tr} \int d^{D} x^{\mu} h^{1 / 2} F_{\mu \nu} F_{\sigma \tau} h^{\mu \sigma} h^{\nu \tau},
$$

can be expanded to give

$$
\begin{aligned}
\mathscr{L}= & -\frac{1}{8} \operatorname{Tr} \int d^{D^{\prime}} x^{i} d^{N^{\prime}} y^{\alpha} h^{1 / 2}\left[F_{i j} F_{k l} h^{i k} h^{j l}\right. \\
& \left.+\frac{2}{R^{2}} F_{i \alpha} F_{j \beta} h^{i j} h^{\alpha \beta}+\frac{1}{R^{4}} F_{\alpha \beta} F_{\gamma \delta} h^{\alpha \beta} h^{\beta \delta}\right] .
\end{aligned}
$$

The components of the field tensor $F_{i j}$ are defined in terms of $A_{i}$ and cannot be simplified. We have expressions for the other components of the field tensor, from (4.25), (4.26). These have not been gauge transformed to their correct form on the coset space, although we know, having applied the constraints, that this can be done. However, yet again we can calculate on the symmetry group. We have

$$
F_{i \alpha}^{a} F_{j \beta}^{a} h^{i j} \xi_{m}^{\alpha} \xi_{m}^{\beta}=F_{\alpha i}^{a} F_{\hat{\beta} j}^{a} \xi_{m}^{\hat{\alpha}} \xi_{m}^{\hat{\beta}} h^{i j},
$$

since the extra components of the field tensor on the r.h.s. all vanish. Now substituting the expressions (4.25) for the field tensor, and using the equality of left and right group metrics together with the orthogonality relations (4.6), we find

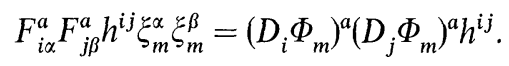

The incorrect gauge does not matter, since we have calculated a gauge invariant 
quantity. In a similar way

$$
\begin{aligned}
F_{\alpha \beta}^{a} F_{\gamma \delta}^{a} h^{\alpha \gamma} h^{\beta \delta} & =\left(f_{r s t} \Phi_{t}+\left[\Phi_{r}, \Phi_{s}\right]\right)^{a}\left(f_{r s t} \Phi_{t}+\left[\Phi_{r}, \Phi_{s}\right]\right)^{a} \\
= & 2 \Phi_{t}^{a} \Phi_{t}^{a}+2 f_{r s t} g^{a b c} \Phi_{t}^{a} \Phi_{r}^{b} \Phi_{s}^{c} \\
& \quad+g^{a b c} g^{a d e} \Phi_{r}^{b} \Phi_{r}^{d} \Phi_{s}^{c} \Phi_{s}^{e} \\
& \equiv 2 V(\Phi) .
\end{aligned}
$$

Apart from the factor $h^{1 / 2}$, the Lagrangian is explicitly independent of $y^{\alpha}$, so integrating we obtain

$$
\begin{aligned}
\mathscr{L}= & \Omega \int d^{D^{\prime}} x R^{N^{\prime}}\left(\operatorname{det} h_{i j}\right)^{1 / 2}\left[\frac{1}{4} F_{i j}^{a} F_{k l}^{a} h^{i k} h^{j l}\right. \\
& \left.+\frac{1}{2 R^{2}}\left(D_{i} \Phi_{m}\right)^{a}\left(D_{j} \Phi_{m}\right)^{a} h^{i j}+\frac{1}{2 R^{4}} V(\Phi)\right],
\end{aligned}
$$

where $\Omega$ is the volume of the coset space. This action is not in its usual canonical form, since it still contains explicit geometrical factors $R(x)$, apart from the metric. We can redefine the metric

$$
g^{i j}=f(x) h^{i j}
$$

in which case we obtain a canonical form if and only if $f=R^{2}$ and $D^{\prime}+N^{\prime}=4$, that is, the original space is 4-dimensional, and then the action is

$$
\begin{aligned}
\mathscr{L}= & C \int d^{D^{\prime}} x g^{1 / 2}\left[\frac{1}{4} F_{i j}^{a} F_{k l}^{a} g^{i k} g^{j l}\right. \\
& \left.+\frac{1}{2}\left(D_{i} \Phi_{m}\right)^{a}\left(D_{j} \Phi_{m}\right)^{a} g^{i j}+V(\Phi)\right],
\end{aligned}
$$

where $C$ is a constant. This will describe an action in curved space even if the original metric $h^{\mu \nu}$ is flat, but the coset space is curved. There is an alternative case of physical interest. We may start in more than four dimensions and use the symmetry to reduce to four, interpreting the reduced Lagrangian as physically relevant. We shall then require that the $h^{i j}$ be simply that of flat space. $R$ must be a constant in this case. The form of the Lagrangian (5.7) is canonical if we rescale $\Phi . R$ will then appear in the ratio of coupling constants to mass parameters in the Higgs potential.

An interesting example, details of which appear in reference [14], seems to be the case of spherical symmetry in 6 dimensions applied to an SU(3) gauge theory, the 2 extra dimensions describing a sphere of radius $R$. One solution, with the largest set of Higgs fields, reduces to the 4-dimensional Weinberg-Salam model without fermions. The Weinberg angle is predicted, as well as the Higgs particle mass, leaving only the e.m. coupling constant and the mass of the $W$ boson as free parameters. This scheme may be theoretically less ad hoc than the conventional reduction from SU(3) theory using a super-strong Higgs mechanism [15], or than the recent scheme of Fairlie which is similar to ours, using toroidal symmetry $\mathrm{U}(1) \times \mathrm{U}(1)$ rather than $\mathrm{SO}(3)$ to reduce from 6 to 4 dimensions, but then requiring an apparently arbitrary ansatz to obtain $S U(2) \times U(1)$ fields rather than SU(3) fields [16].

Apart from the example of Witten's, which we examine in the next section, 
we have considered one other example of reduction from 4-dimensions. This is the case of $\mathrm{SO}(4)$ symmetry in an $\mathrm{SU}(2)$ gauge theory. It is simple to show, using our methods, that the action reduces to a one-dimensional scalar field theory with no residual gauge freedom.

Finally, we mention a feature of the Lagrangian (5.7) which is very intriguing, namely, the global invariance of the Higgs potential, and of the kinetic terms for the Higgs fields, under the group $S$. This is in the algebraic sense of a flavour group and seems disconnected from the geometrical way in which $S$ has previously been considered. This symmetry is also broken when the constraints are applied, down to the little group of $R$ in $S$.

\section{Witten's Ansatz Re-Examined}

To illustrate our formal machinery, we rederive Witten's ansatz, where both the gauge and symmetry groups are $\mathrm{SO}(3)$. The $\mathrm{SO}(3)$ symmetry group is paramatrized by the three Euler angles $(\chi, \theta, \varphi)$, and a general group element $s(\chi, \theta, \varphi)$ is defined as

$$
s(\chi, \theta, \varphi)=\mathscr{R}_{z}(\chi) \mathscr{R}_{x}(\theta) \mathscr{R}_{z}(\varphi),
$$

where $\mathscr{R}_{z}(\chi)$ denotes a rotation about the $z$-axis through an angle $\chi$, etc. Since rotations about the $z$-axis form an $\mathrm{SO}(2)$ subgroup we can compare (6.1) with the general expression (3.10) and conclude that the angles $\theta, \varphi$ are coordinates for the right coset space $\mathrm{SO}(3) / \mathrm{SO}(2)$, with $\chi$ the coordinate of $\mathrm{SO}(2)$. The Witten ansatz is defined on a four-dimensional space with coordinates $(t, r, \theta, \varphi)$ where the spherical polar angles are to be identified with the coset space coordinates. The symmetry generators, written as $\xi_{m}^{\alpha}=\left(\xi_{m}^{\theta}, \xi_{m}^{\varphi}\right)$ are

$$
\begin{aligned}
& \xi_{1}^{\alpha}=(\cos \varphi,-\operatorname{ctg} \theta \sin \varphi), \\
& \xi_{2}^{\alpha}=(-\sin \varphi,-\operatorname{ctg} \theta \cos \varphi), \\
& \xi_{3}^{\alpha}=(0,1) .
\end{aligned}
$$

These may be obtained, by projection, from the infinitesimal right translations on the symmetry group, written $\xi_{m}^{2}=\left(\xi_{m}^{\chi}, \xi_{m}^{\theta}, \xi_{m}^{\varphi}\right)$,

$$
\begin{aligned}
& \xi_{1}^{\hat{\alpha}}=\left(\frac{\sin \varphi}{\sin \theta}, \cos \varphi,-\operatorname{ctg} \theta \sin \varphi\right), \\
& \xi_{2}^{\hat{\alpha}}=\left(\frac{\cos \varphi}{\sin \theta},-\sin \varphi,-\operatorname{ctg} \theta \cos \varphi\right), \\
& \xi_{3}^{\hat{\alpha}}=(0,0,1),
\end{aligned}
$$

Both sets of generators satisfy the SO(3) Lie bracket algebra

$$
\left[\xi_{m}, \xi_{n}\right]=\varepsilon_{m n p} \xi_{p}
$$

and in both sets we have suppressed the components $\xi_{m}^{r}, \xi_{m}^{t}$ which vanish.

To construct the symmetric gauge fields we use the symmetry generators 
on the group $\tilde{\xi}_{m \hat{\alpha}}$, which are

$$
\begin{aligned}
& \tilde{\xi}_{1 \hat{\alpha}}=(0,-\cos \chi,-\sin \theta \sin \chi), \\
& \tilde{\xi}_{2 \hat{\alpha}}=(0,-\sin \chi, \sin \theta \cos \chi), \\
& \tilde{\xi}_{3 \hat{\alpha}}=(-1,0,-\cos \theta) .
\end{aligned}
$$

The generator of the subgroup $\mathrm{SO}(2)$, which defines the sphere as a coset space, is $T^{3}$, so we must impose the constraints

$$
\begin{aligned}
& \partial_{i} \Phi_{3}^{a}-\varepsilon^{a b c} A_{i}^{b} \Phi_{3}^{c}=0, \\
& \varepsilon_{m 3 p} \Phi_{p}^{a}+\varepsilon^{a b c} \Phi_{m}^{b} \Phi_{3}^{c}=0 .
\end{aligned}
$$

Since the little group of $\Phi_{3}$ is U(1) we expect an abelian Higgs theory to emerge. Fixing a gauge where $\Phi_{3}^{1}=\Phi_{3}^{2}=0$, the solution of the constraint equations is

$$
\begin{aligned}
A_{i}^{a} & =\left(0,0, A_{i}\right), \\
\Phi_{1}^{a} & =\left(\phi_{1}, \phi_{2}, 0\right), \\
\Phi_{2}^{a} & =\left(\phi_{2},-\phi_{1}, 0\right), \\
\Phi_{3}^{a} & =(0,0,1),
\end{aligned}
$$

where $\phi_{1}, \phi_{2}$ and $A_{i}$ are arbitrary functions of $(r, t)$. The components of the gauge fields $A_{\hat{\alpha}}^{a}=\Phi_{m}^{a} \tilde{\xi}_{m \hat{\alpha}}$ are now

$$
\begin{aligned}
& A_{\chi}^{a}=(0,0,-1), \\
& A_{\theta}^{a}=\left(-\phi_{1} \cos \chi-\phi_{2} \sin \chi,-\phi_{2} \cos \chi+\phi_{1} \sin \chi, 0\right), \\
& A_{\varphi}^{a}=\left(-\phi_{1} \sin \theta \sin \chi+\phi_{2} \sin \theta \cos \chi,-\phi_{2} \sin \theta \sin \chi-\phi_{1} \sin \theta \cos \chi,-\cos \theta\right) .
\end{aligned}
$$

We see that $A_{\chi}$ is non-zero, and that $A_{\theta}$ and $A_{\varphi}$ depend on $\chi$, but after a gauge rotation about the 3 rd isoaxis through an angle $\chi$, leaving $A_{i}$ unchanged, we find that $A_{\chi}$ vanishes and

$$
\begin{aligned}
A_{t}^{a} & =\left(0,0, A_{0}\right), \\
A_{r}^{a} & =\left(0,0, A_{1}\right), \\
A_{\theta}^{a} & =\left(-\phi_{1},-\phi_{2}, 0\right), \\
A_{\varphi}^{a} & =\left(\phi_{2} \sin \theta,-\phi_{1} \sin \theta,-\cos \theta\right),
\end{aligned}
$$

which agrees with Witten's result.

Knowing this gauge rotation, we can use the result $(4.35)$ to calculate $W_{m}$. We find

$$
\begin{aligned}
& W_{1}^{a}=\left(0,0, \frac{\sin \varphi}{\sin \theta}\right), \\
& W_{2}^{a}=\left(0,0, \frac{\cos \varphi}{\sin \theta}\right), \\
& W_{3}^{a}=0 .
\end{aligned}
$$


These expressions and those above for the gauge field can also be simply obtained from (4.36) and (4.37). It can be checked that on the original space

$$
L_{\xi_{m}} A_{\mu}=D_{\mu} W_{m} \text {. }
$$

We have rederived Witten's ansatz and proved that it is essentially unique, as he stated. In fact there are degenerate cases which he ignored. The solution of the constraint equations (6.6) and (6.7) where $\phi_{1}, \phi_{2}$ vanish, but $\Phi_{3}^{3}$ is an arbitrary constant, leads to an abelian monopole of arbitrary charge, with a radial electric field. The solution where all $\Phi_{m}$ vanish gives a pure SU (2) gauge theory after dimensional reduction.

The flat space metric in spherical polars is of the general form (5.1), required for dimensional reduction of the action, and the 2 dimensional action that Witten obtained is an example of our general result.

\section{Conclusions}

We have developed a general method for finding gauge fields invariant under any group of symmetries and for any gauge group. The symmetries imply that the space on which the gauge fields are defined can be decomposed into a family of subspaces, each of which can be identified with a coset space of the symmetry group. We have shown that symmetric gauge fields on this coset space can be lifted to the group itself, where the gauge group decouples, and the fields may be expressed in terms of infinitesimal translations on the symmetry group. Certain purely algebraic constraint equations have to be satisfied in order to obtain a well-defined solution on the coset space.

Scalar fields appear naturally as a result of the symmetries, and the pure gauge theory reduces to a lower dimensional Higgs model with a precisely determined quartic potential. In practice, it is easier to calculate the Lagrangian for this Higgs model than the explicit form for the symmetric gauge fields.

\section{Appendix}

We analyse here the second constraint, Eq. (4.31), which must be imposed to obtain a well defined symmetric gauge field on the coset $S / R$. It has been assumed already that the generators $J^{n}$ and $-\Phi_{n}^{a} T^{a}$, for $n>N^{\prime}$ in each case, both generate $R$, as a subgroup of $S$ and $G$ respectively.

The constraint (4.31)

$$
f_{m n p} \Phi_{p}^{a}+g^{a b c} \Phi_{m}^{b} \Phi_{n}^{c}=0, \quad \forall m, \forall n>N^{\prime},
$$

can be rewritten as

$M_{m p}^{\prime} \Phi_{p}^{a}=\Phi_{m}^{b} M^{\prime \prime b a}$,

where $M^{\prime}$ and $M^{\prime \prime}$ are matrix representations of the Lie algebra of $R$, with

$$
\begin{array}{ll}
M^{\prime}\left(J^{n}\right)_{m p}=-f_{n m p}, & \forall n>N^{\prime}, \\
M^{\prime \prime}\left(J^{n}\right)^{b a}=-\Phi_{n}^{c} g^{c b a}, & \forall n>N^{\prime} .
\end{array}
$$


$M^{\prime}$ reduces naturally into two representations: the adjoint representation of $R$ together with the induced representation of $R$ over $S / R$. We have solved the part of the problem involving the adjoint representation of $R$ by assuming that $R$ is a subgroup of $G$, so now we need only consider the restriction of (A.2) to the coset space. There is no restriction on $M^{\prime \prime}$, which is the induced representation of $R$ over $G$. We can choose bases so that both representations split into their irreducible parts, and such that the submatrices of any irreducible component occurring more than once are identical. The irreducible components of $M^{\prime}$ are denoted by $M_{i}^{\prime}$, and those of $M^{\prime \prime}$ by $M_{i}^{\prime \prime}$.

Now consider, for some pair $\left(M_{i}^{\prime}, M_{j}^{\prime \prime}\right)$ the restriction of Eq. (A.2) to the subspaces on which this pair acts, which we write

$$
\left(M_{i}^{\prime}\right)_{m p} \Phi_{p}^{(i j) a}=\Phi_{m}^{(i j) b}\left(M_{j}^{\prime \prime}\right)^{b a} .
$$

If the matrix $\Phi^{(i j)}$ is non-vanishing, it is necessary that its rows be independent, and also its columns, since otherwise either $M_{i}^{\prime}$ or $M_{j}^{\prime \prime}$ will be reducible. If $M_{i}^{\prime}$ and $M_{j}^{\prime \prime}$ have different dimensions, this means that $\Phi^{(i j)}$ must be zero. Even if $M_{i}^{\prime}$ and $M_{j}^{\prime \prime}$ are different irreducible representations of the same dimension, $\Phi^{(i j)}$ vanishes, since (A.4) implies that $M_{i}^{\prime}$ and $M_{j}^{\prime \prime}$ are related by a change of basis. Finally, if $M_{i}^{\prime}$ and $M_{j}^{\prime \prime}$ are the same representation, with identical matrices, then (A.4) states that $\Phi^{(i j)}$ commutes with all matrices in the representation $M_{i}^{\prime}$. Schur's lemma then requires

$$
\Phi^{(i j)}=\phi^{(i j)}(x) \mathbf{1},
$$

where $\phi^{(i j)}(x)$ is an arbitrary function.

We conclude that for each irreducible pair $\left(M_{i}^{\prime}, M_{j}^{\prime \prime}\right)$ there remains a single Higgs field component $\phi^{(i j)}$, associated with a unit matrix, if $M_{i}^{\prime}$ and $M_{j}^{\prime \prime}$ are the same. All other Higgs field components must vanish.

Acknowledgements. We would like to thank M. F. Atiyah for encouraging an investigation of this problem. We are grateful to several colleagues at ENS, and also to R. Kerner, for discussions.

We thank the French Government for research fellowships.

Note added. After this article was completed we discovered that the construction of invariant connections on principal fibre bundles, equivalent to our symmetric gauge fields, has been considered in the mathematical literature [17], [18].

\section{References}

1. 't Hooft, G. : Nucl. Phys. B79, 276-284 (1974)

Polyakov, A. M. : JETP Lett. 20, 194-195 (1974)

2. Belavin, A. A., Polyakov, A. M., Schwartz, A., Tyupkin, Y. : Phys. Lett. 59B, 85-87 (1975)

3. Witten, E. : Phys. Rev. Lett. 38, 121-124 (1977)

4. Corrigan, E., Olive, D., Fairlie, D. B., Nuyts, J. : Nucl. Phys. B106, 475-492 (1976)

Goddard, P., Olive, D. I. : Rep. Prog. Phys. 41, 1357-1437 (1978)

5. Choquet-Bruhat, Y., De Witt-Morette, C., Dillard-Bleick, M. : Analysis, Manifolds and Physics. Amsterdam : North Holland 1977 
6. Bergmann, P. G., Flaherty, E. J. : J. Math. Phys. 19, 212-214 (1978)

7. Harnad, J., Shnider, S., Vinet, L. : J. Math. Phys. 20, 931-942 (1979)

8. Scherk, J., Schwarz, J. : Nucl. Phys. B81, 118-144 (1974)

9. Cremmer, E., Scherk, J. : Nucl. Phys. B118, 61-75 (1977)

Luciani, J. F. : Nucl. Phys. B135, 111-130 (1978)

Horváth, Z., Palla, L., Cremmer, E., Scherk, J. : Nucl. Phys. B127, 57-65 (1977)

Forgács, P., Horváth, Z. : ITP preprints Budapest n ${ }^{\circ} 384$ and 385 (1978)

10. Gliozzi, F., Scherk, J., Olive, D. : Nucl. Phys. B122, 253-290 (1977)

Cremmer, E., Julia, B. : Phys. Lett. 80B, 48-51 (1978)

Scherk, J., Schwarz, J. : Nucl. Phys. B153, 61-88 (1979)

11. Lohe, M. : Phys. Lett. 70B, 325-328 (1977)

12. Olive, D. : Nucl. Phys. B153, 1-12 (1979)

13. Manton, N. S. : Nucl. Phys. B126, 525-541 (1977)

14. Manton, N. S. Nucl. Phys. B158, 141-153 (1979)

15. Weinberg, S. : Phys. Rev. D5, 1962-1967 (1971)

16. Fairlie, D. : Phys. Lett. 82B, 97-100 (1979)

17. Kobayashi, S., Nomizu, K. : Foundations of differential geometry. Vol. 1. New York : Interscience 1963

18. Wang, H-C. : Nagoya Math. J. 13, 1-19(1958)

Communicated by E. Brézin

Received April 30, 1979 
